\title{
Lyapunov Exponents and Uniform Weak Normally Repelling Invariant Sets
}

\author{
Paul Leonard Salceanu and Hal L. Smith \\ School of Mathematical and Statistical Sciences, Arizona State University, Tempe, \\ AZ 85287, USA, salceanu@mathpost. asu.edu and halsmith@asu.edu
}

\begin{abstract}
Let $M$ be a compact invariant set contained in a boundary hyperplane of the positive orthant of $\mathbb{R}^{n}$ for a discrete or continuous time dynamical system defined on the positive orthant. Using elementary arguments, we show that $M$ is uniformly weakly repelling in directions normal to the boundary in which $M$ resides provided all normal Lyapunov exponents are positive. This result is useful in establishing uniform persistence of the dynamics.
\end{abstract}

\section{Introduction}

Dynamical systems models in population biology are typically defined on the nonnegative cone in Euclidean space. In order to establish persistence of some or all components (species) in the model, it is often necessary to show that a compact invariant set on the boundary of the cone is an isolated invariant set and that it is repelling, at least in some directions normal to $M$. See [4, $5,15,19,20,7]$ for recent work in the theory of persistence, sometimes called permanence. In this paper, building on the work of $[4,15]$ and $[13,14]$, we show that Lyapunov exponents can be used to establish the requisite repelling properties for both discrete and continuous time systems. This is well known when $M$ is a fixed point or periodic orbit but not so when the dynamics on $M$ is more complicated. We use only elementary arguments rather than appealing to the multiplicative ergodic theorem $[1,2,4,15]$. This extends our earlier work in $[11,12,13]$ which covered only the discrete case.

The use of Lyapunov exponents in the study of biological models was pioneered by Metz [9], Metz et. al. [8], who proposed that the dominant Lyapunov exponent gives the best measure of invasion fitness, and by Rand et. al. [10] who used it to characterize the invasion "speed" of a rare species. See also the more recent review by Ferriere and Gatto [3] which deals with computational aspects. Roughly, a positive dominant Lyapunov exponent corresponding to a potential invading species in the environment set by a resident species attractor implies that the invader can successfully invade. Our results will give 
a mathematically rigorous interpretation of this for the nonlinear dynamics. Ashwin et al. [2] use "normal" Lyapunov exponents and invariant measures to answer the following question: if $f: M \rightarrow M$ is a smooth map on a smooth finite dimensional manifold, $N$ is a lower dimensional submanifold for which $f(N) \subseteq N$, and $A \subseteq N$ is an attractor for $f_{\mid N}$, is $A$ an attractor for $f$, or it is an unstable saddle?

\section{Main Results}

Due to our need to use both subscripts and occasionally superscripts for sequences, we adopt the notation $x=\left(x^{(1)}, \ldots, x^{(m)}\right)^{T} \in \mathbb{R}^{m}$. Let $|x|=\sum\left|x^{(i)}\right|$ denote the norm on $\mathbb{R}^{m}$ and $d(z, M)$ for the distance of $z \in \mathbb{R}^{m}$ to $M \subset \mathbb{R}^{m}$. Denote by $\mathbb{R}_{+}^{m}=\left\{x \in \mathbb{R}^{m}: x^{(i)} \geq 0, \forall i\right\}$ the nonnegative cone in $\mathbb{R}^{m}$. We write $x \geq 0$ when $x \in \mathbb{R}_{+}^{m}$; if $A=\left(a_{i j}\right)$ is an $n \times n$ matrix, then $A \geq 0$ if $a_{i j} \geq 0$ for all $i, j$. Observe that $|x+y|=|x|+|y|$ for vectors $x, y \in \mathbb{R}_{+}^{m}$. We let $Z_{+}=\mathbb{R}_{+}^{m}$.

We consider the discrete dynamical system

$$
z_{n+1}=F\left(z_{n}\right), z_{0} \in Z_{+}
$$

and the continuous dynamical system

$$
z^{\prime}(t)=F(z(t)), z_{0}:=z(0) \in Z_{+}
$$

on the nonnegative cone $Z_{+}$.

It is assumed that (1) and (2) generate a semi-dynamical system on $Z_{+}$. In case of (1), $F: Z_{+} \rightarrow Z_{+}$is continuous; in case (2), $F: Z_{+} \rightarrow \mathbb{R}^{m}$ satisfies $z \in Z_{+}, z^{(i)}=0 \Rightarrow F^{(i)}(z) \geq 0$ and sufficient regularity properties such that solutions of (2) exist and are unique.

We assume that $m=p+q, p, q \geq 1$ and that $Z_{+}$is decomposed as follows

$$
z=(x, y) \in Z_{+} \equiv \mathbb{R}_{+}^{m}=\mathbb{R}_{+}^{p} \times \mathbb{R}_{+}^{q}
$$

Compatible with this decomposition, assume that $F(z)=(f(z), g(z))$. Define

$$
X=\left\{z=(x, y) \in Z_{+}: y=0\right\} .
$$

We assume:

$$
X \text { and } Z_{+} \backslash X \text { are positively invariant sets. }
$$

Positive invariance of $X$ for both (1) and (2) means that $g(x, 0)=0,(x, 0) \in$ $X$.

If $F$ satisfies additional smoothness hypotheses, then it would follow from positive invariance of $X$ that (1) and (2) can be expressed as

$$
\left\{\begin{array}{l}
x_{n+1}=f\left(z_{n}\right) \\
y_{n+1}=A\left(z_{n}\right) y_{n}
\end{array}\right.
$$


and, respectively as

$$
\left\{\begin{array}{l}
x^{\prime}=f(z) \\
y^{\prime}=A(z) y
\end{array}\right.
$$

where the matrix function $z \rightarrow A(z)$ is continuous and satisfies:

$$
A(z) \geq 0, \quad z \in X
$$

in case of (4), and

$$
A_{i j}(z) \geq 0, i \neq j, \quad z \in X
$$

in case of (5). Rather than assume the required smoothness of $F$, we simply assume hereafter that (6) holds for (4) and that (7) holds for (5).

Let $\mathbb{T}_{+}$denote either $\mathbb{Z}_{+}$or $\mathbb{R}_{+}$. When we write $t \in \mathbb{T}_{+}$, that means we consider both discrete and continuous cases. To make the notation more general, let also $z_{t}, y_{t}$ etc. denote $z(t), y(t)$ etc., when $t \in \mathbb{R}_{+}$.

Let $(\phi(t))_{t \in \mathbb{T}_{+}}$be the dynamical system generated by (4) (for $t \in \mathbb{Z}_{+}$) or by (5) (for $t \in \mathbb{R}_{+}$), respectively. Let $\mathcal{O}^{+}(z):=\left\{\phi(t, z): t \in \mathbb{T}_{+}\right\}$, which we will refer to as the positive orbit through $z$. Let $I$ denote the identity matrix.

Let $P(n, z)$ and $P(t, z)$ denote the fundamental matrix solutions for

$$
u_{n+1}=A(\phi(n, z)) u_{n}
$$

and for

$$
v^{\prime}(t)=A(\phi(t, z)) v(t)
$$

They satisfy:

$$
P(n+1, z)=A(\phi(n, z)) P(n, z), P(0, z)=I
$$

for discrete time and

$$
\frac{d}{d t} P(t, z)=A(\phi(t, z)) P(t, z), P(0, z)=I
$$

for continuous time. In either case, it follows from (6), (7) that

$$
P(t, z) \geq 0, \forall z \in X, \forall t \in \mathbb{T}_{+} .
$$

Let $M \subseteq X$ be compact and positively invariant set. We envision that in typical applications, $M$ will be an invariant set in the interior of the face $X$ of the cone $Z_{+}$. In this paper, we will focus on the behavior of solutions near $M$ in $Z_{+} \backslash X$.

Following Arnold [1], $P(n, z)$ (or $P(t, z)$ ) is a matrix co-cycle generated by (8) (or by (9)). It is trivial to check that $P$ has the following (co-cycle) property:

$$
P\left(t_{2}, \phi\left(t_{1}, z\right)\right) P\left(t_{1}, z\right)=P\left(t_{1}+t_{2}, z\right), \forall z \in Z_{+}, \forall t_{1}, t_{2} \in \mathbb{T}_{+} .
$$

Hereafter, when we take $t \in \mathbb{Z}_{+}$, we refer to (4), and when we take $t \in \mathbb{R}_{+}$, we refer to (5). 
Following $[1,2,6]$, for any $z \in M$ and $\eta \in \mathbb{R}^{q}$ we define the normal Lyapunov exponent $\lambda(z, \eta)$ as

$$
\lambda(z, \eta)=\limsup _{t \rightarrow \infty} \frac{1}{t} \ln |P(t, z) \eta|, t \in \mathbb{T}_{+} .
$$

As noted in [1], $\lambda(z, \eta) \in\{-\infty\} \cup \mathbb{R}$ and $\lambda(z, \eta)=\lambda(z, a \eta), \forall a \in \mathbb{R} \backslash\{0\}$. We only consider the case that $\eta \in \mathbb{R}_{+}^{q}$ because in that case $z+(0, \eta) \in Z_{+}$and $(0, \eta)$ represents a normal vector to $M$ at $z$. The co-cycle property (13) can be used to show that

$$
\lambda(z, \eta)=\lambda(\phi(s, z), P(s, z) \eta), s \geq 0 .
$$

Definition 1. We call the compact positively invariant set $M$ a uniformly weak normally repelling set if there exists $\varepsilon>0$ such that

$$
\limsup _{t \rightarrow \infty} d(\phi(t, z), M)>\epsilon, \forall z \in Z_{+} \backslash X .
$$

Equivalently, in view of (3), there exists a neighborhood $V$ of $M$ in $Z_{+}$such that

$$
\forall z \in V \backslash X, \exists t=t(z)>0, \phi(t, z) \notin V .
$$

We stress that $M$ may be an attractor relative to the dynamics restricted to the positively invariant set $X$ but we are concerned with the behavior of solutions near $M$ in the positively invariant set $Z_{+} \backslash X$. In [13], we used the terminology " $M$ a uniformly weak repeller" for the definition above; we believe the current terminology gives a more accurate description. The adjective "uniform" reflects that $\epsilon$ is independent of $z$; "weak" reflects that limit superior, rather than limit inferior, appears in the definition; "normal" indicates that we are only interested in the behavior of solutions in $Z_{+} \backslash X$.

First, we give a lemma adapted from $[13,14]$ that gives an alternative formulation for the "positivity" of Lyapunov exponents. Let

$$
U=\left\{\eta \in \mathbb{R}_{+}^{q}:|\eta|=1\right\} .
$$

Lemma 1. Let $K \subset X$ be compact. Assume that

$$
\forall(z, \eta) \in K \times U, \exists \mathbb{T}_{+} \backslash\{0\} \ni \tau=\tau(z, \eta) \text { such that }|P(\tau, z) \eta|>1 .
$$

Then $\exists c>1, \exists V$ a bounded neighborhood of $K$ in $Z_{+}$, such that if $L \subseteq V$ is a positively invariant set, then $L \subset X$ and

$$
\forall(z, \eta) \in L \times U, \exists\left(\nu_{p}\right)_{p} \subseteq \mathbb{T}_{+}, \nu_{p} \rightarrow \infty,\left|P\left(\nu_{p}, z\right) \eta\right|>c^{p}, \forall p \geq 1 .
$$

If, in addition, $K$ is positively invariant, then (16) is equivalent to

$$
\lambda(z, \eta)>0, \forall(z, \eta) \in K \times U .
$$


Proof. Let $W=K \times U$ (so $W$ is compact) and $\hat{w}=(\hat{z}, \hat{\eta}) \in K \times U$. From (16) we have that there exists $\hat{\tau}=\hat{\tau}(\hat{z}, \hat{\eta}) \in \mathbb{T}_{+} \backslash\{0\}$ such that $|P(\hat{\tau}, \hat{z}) \hat{\eta}|>1$. The function $(z, \eta) \mapsto|P(\hat{\tau}, z) \eta|$ being continuous, there exist $\delta_{\hat{w}}>0, c_{\hat{w}}>1$ such that

$$
|P(\hat{\tau}, z) \eta|>c_{\hat{w}}, \forall w=(z, \eta) \in B_{\delta_{\hat{w}}}(\hat{w}):=\left\{\tilde{w} \in Z_{+} \times U|| \tilde{w}-\hat{w} \mid<\delta_{\hat{w}}\right\} .
$$

Since $W$ is compact, there exists a finite set $\left\{w^{1}, \ldots, w^{k}\right\} \subseteq W$ such that $W \subset C:=\cup_{i=1}^{k} B_{\delta_{w^{i}}}\left(w^{i}\right)$, where for every $i=1, \ldots, k, \delta_{w^{i}}$ is the quantity corresponding to $w^{i}$, coming from (19) (i.e., for every $i=1, \ldots, k,(19)$ is satisfied with $\hat{w}$ replaced by $\left.w^{i}\right)$. To simplify notation, let $\tau_{i}:=\tau\left(w^{i}\right), \delta_{i}:=$ $\delta_{w^{i}}, i=1, \ldots, k$. Also, let $c:=\min _{i} c_{w^{i}}$ (hence $c>1$ ) and $\tau=\max _{i} \tau_{i}$. Thus, from (19) we have that

$$
\left|P\left(\tau_{i}, z\right) \eta\right|>c, \forall w=(z, \eta) \in B_{\delta_{i}}\left(w^{i}\right), \forall i=1, \ldots, k .
$$

Now let $V \subset Z_{+}$be a bounded neighborhood of $K$ such that $V \times U \subseteq C$ and let $L \subseteq V$ be positively invariant. We prove that $L \subset X$ arguing by contradiction: suppose $L \backslash X \neq \emptyset$. Let $a=\left(a_{x}, a_{y}\right) \in L \backslash X$. Since $\left|a_{y}\right|>0$, we can define $\alpha:=a_{y} /\left|a_{y}\right|$. Note that $\alpha \in U$. We will show that

$$
\exists\left(\nu_{p}\right)_{p} \subset \mathbb{T}_{+}, \nu_{p} \rightarrow \infty, \text { such that }\left|P\left(\nu_{p}, a\right) \alpha\right|>c^{p}, \forall p \geq 1 .
$$

by inductively constructing the sequence $\left(\nu_{p}\right)_{p}$. Thus, there exists $i \in\{1, \ldots, k\}$ such that $(a, \alpha) \in B_{\delta_{i}}\left(w^{i}\right)$. Then, from (20) we have $\left|P\left(\nu_{1}, a\right) \alpha\right|>c$, where $\nu_{1}=\tau_{i}$. Now suppose $\left|P\left(\nu_{p}, a\right) \alpha\right|>c^{p}$ for some $p \geq 1$. Let $\tilde{\alpha}=$ $P\left(\nu_{p}, a\right) \alpha /\left|P\left(\nu_{p}, a\right) \alpha\right|$. Since $L \backslash X$ is positively invariant, $\phi\left(\nu_{p}, a\right) \in L \backslash X$, hence $\phi^{(2)}\left(\nu_{p}, a\right)>0$, where $\phi^{(2)}(t, z)$ denotes the vector formed with the last $q$ components of $\phi(t, z)$. So

$$
P\left(\nu_{p}, a\right) \alpha=\frac{1}{\left|a_{y}\right|} P\left(\nu_{p}, a\right) a_{y}=\frac{1}{\left|a_{y}\right|} \phi^{(2)}\left(\nu_{p}, a\right)>0 .
$$

Thus, $\tilde{\alpha} \in U$. There exists $j \in\{1, \ldots, k\}$ such that $\left(\phi\left(\nu_{p}, a\right), \tilde{\alpha}\right) \in B_{\delta_{j}}\left(w^{j}\right)$. Then again, from (20) we have

$$
\left|P\left(\tau_{j}, \phi\left(\nu_{p}, a\right)\right) \tilde{\alpha}\right|>c, \text { which implies }\left|P\left(\tau_{j}, \phi\left(\nu_{p}, a\right)\right) P\left(\nu_{p}, a\right) \alpha\right|>c^{p+1} .
$$

This means, using (13), that $\left|P\left(\nu_{p+1}, a\right) \alpha\right|>c^{p+1}$, where we define $\nu_{p+1}=$ $\nu_{p}+\tau_{j}$. Note that, by construction, $\nu_{p} \rightarrow \infty$ as $p \rightarrow \infty$. Hence (21) holds. Then, we have that

$$
\left|\phi^{(2)}\left(\nu_{p}, a\right)\right|=\left|P\left(\nu_{p}, a\right) a_{y}\right|>c^{p}\left|a_{y}\right|, \forall p \geq 1,
$$

which implies that $\left|\phi^{(2)}\left(\nu_{p}, a\right)\right| \rightarrow \infty$ as $p \rightarrow \infty$. But this is a contradiction to $L$ being bounded. Hence, $L \subset X$.

Now (17) can be proved identically as for (21), using that $L \subset X$ is positively invariant and that $P(t, z) \geq 0, \forall z \in X, \forall t \in \mathbb{T}_{+}$. 
Now assume that $K$ is also positively invariant. The implication $(18) \Rightarrow$ (16) is trivial. For the converse, using (17) and the fact that $\nu_{p} \leq p \tau, \forall p \geq 1$, we have, for all $(z, \eta) \in K \times U$, that

$$
\left|P\left(\nu_{p}, z\right) \eta\right|^{1 / \nu_{p}}>c^{p / \nu_{p}} \geq c^{1 / \tau} \Rightarrow \frac{1}{\nu_{p}} \ln \left|P\left(\nu_{p}, z\right) \eta\right|>\frac{1}{\tau} \ln c, \forall p \geq 1 .
$$

Hence

$$
\lambda(z, \eta)=\limsup _{t \rightarrow \infty} \frac{1}{t} \ln (|P(t, z) \eta|) \geq \frac{1}{\tau} \ln c>0 .
$$

This completes our proof.

In the next result we establish sufficient conditions for $M$ to be a uniformly weak normally repelling set. Let

$$
\Omega(M)=\cup_{z \in M} \omega(z),
$$

where $\omega(z)$ represents the omega limit set of $z$.

Theorem 1. Let $M \subset X$ be a nonempty compact and positively invariant. $M$ is a uniformly weak normally repelling set if

$$
\lambda(z, \eta)>0, \forall(z, \eta) \in M \times U .
$$

If

$$
\forall(z, \eta) \in M \times U, \forall t \in \mathbb{T}_{+}, P(t, z) \neq 0
$$

and

$$
\lambda(z, \eta)>0, \forall(z, \eta) \in \Omega(M) \times U
$$

then (23) holds.

Proof. First we show that (23) implies that $M$ is a uniformly weak normally repelling set. For this, we argue by contradiction: suppose $M$ is not a uniformly weak normally repelling set. So, there exists a sequence $\left(\tilde{z}^{m}\right)_{m} \subseteq Z_{+} \backslash X$ such that

$$
\limsup _{t \rightarrow \infty} d\left(\phi\left(t, \tilde{z}^{m}\right), M\right)<1 / m, \forall m \geq 1 .
$$

Hence there exists a sequence $\left(\tau_{m}\right)_{m} \subset \mathbb{T}_{+}$such that, for each $m \geq 1$, we have

$$
d\left(\phi\left(t, \tilde{z}^{m}\right), M\right)<1 / m, \forall t \geq \tau_{m} .
$$

Let $z^{m}=\left(x^{m}, y^{m}\right)=\phi\left(\tau_{m}, \tilde{z}^{m}\right)$. Using the positive invariance of $Z_{+} \backslash X$, we have that

$$
y^{m}>0, \forall m \geq 1 \text {. }
$$

From the semiflow property of $\phi$ and from (26) we get

$$
d\left(\phi\left(t, z^{m}\right), M\right)<1 / m, \forall t \in \mathbb{T}_{+}, m \geq 1 .
$$


Using (23), we obtain from Lemma 1 (applied with $K=M$ ) that there exists $V$ a bounded neighborhood of $M$ in $Z_{+}$, having the property that any positively invariant set contained in $V$ is a subset of $X$. Then there exists $m \in \mathbb{N}$ such that $B_{m}:=\left\{z \in Z_{+} \mid d(z, M) \leq 1 / m\right\}$ is contained in $V$. The set $L=\left\{\phi\left(n, z^{m}\right) \mid n \geq 0\right\}$ is positively invariant and, according to (28), it is contained in $B_{m}$. Also (see (27)) $L \backslash X \neq \emptyset$. But this is a contradiction, according to Lemma 1 . Hence, $M$ is a uniformly weak repeller.

Now we prove the final assertion. Let $(a, \alpha) \in M \times U$. Using 2) and the fact that $\omega(a) \subset X$ is compact and invariant, we can again apply Lemma 1 , now with $K=\omega(a)$. So let $V_{a}$ be a neighborhood of $\omega(a)$ and $c>1$ as in the above mentioned lemma. Since $\phi(t, a) \rightarrow \omega(a)$ as $t \rightarrow \infty$, there exists $\tau_{a} \in \mathbb{T}_{+}$such that $\phi(t, a) \in V_{a}, \forall t \geq \tau_{a}$. Let $L=\left\{\phi(t, a) \mid t \geq \tau_{a}\right\}$. Then $L$ is a positively invariant set contained in $V_{a}$. Let $\tilde{\alpha}=P\left(N_{a}, a\right) \alpha /\left|P\left(N_{a}, a\right) \alpha\right|$. Note that $\tilde{\alpha}$ is well defined, due to (24), and that $\tilde{\alpha} \in U$. So, from (17), there exists a sequence $\nu_{p} \rightarrow \infty$ such that $\left|P\left(\nu_{p}, \phi\left(\tau_{a}, a\right)\right) \tilde{\alpha}\right|>c^{p}, \forall p \geq 1$. Thus, using (13) we get

$$
\left|P\left(\nu_{p}+\tau_{a}, a\right) \alpha\right|>c^{p}\left|P\left(\tau_{a}, a\right) \alpha\right|, \forall p \geq 1 .
$$

We can find a $p$ large enough such that to have $c^{p}\left|P\left(\tau_{a}, a\right) \alpha\right|>1$. So, we proved that

$$
\forall(z, \eta) \in M \times U, \exists \tau \in \mathbb{T}_{+} \backslash\{0\} \text { such that }|P(\tau, z) \eta|>1,
$$

which is equivalent to (23), by Lemma 1. This completes our proof.

Note that (24) is automatically satisfied in the continuous case. In the discrete case, it is equivalent to $A(z) \eta \neq 0, \forall z \in M, \forall \eta \in U$.

As it will be seen below, when the matrix $A(z)$ satisfies stronger positivity conditions, then the Lyapunov exponents are independent of the unit vector $\eta$. Let $\| A||=\sup \{|A \xi|:|\xi|=1\}$ denote the norm of an $n \times n$ matrix. For matrices $A, B$, we write $A \leq B$ if $a_{i j} \leq b_{i j}, \forall i, j$; inequality $A \gg 0$ means all entries of $A$ are positive.

Proposition 1. Let $z \in X$ have compact orbit closure $\overline{\mathcal{O}^{+}(z)}$. In the discrete case, assume that

$$
\exists N, P\left(n, z_{0}\right) \gg 0, n \geq N, z_{0} \in \overline{\mathcal{O}^{+}(z)} .
$$

In the continuous case, assume that

$$
A\left(z_{0}\right) \text { is irreducible, } z_{0} \in \overline{\mathcal{O}^{+}(z)} .
$$

Then

$$
\lambda(z, \eta)=\limsup _{t \rightarrow \infty} \frac{1}{t} \ln \|P(t, z)\|, \forall \eta \in U .
$$

In particular, if (29), respectively (30), holds for each $z \in M$, then (31) holds for every $z \in M$. 
Proof. First we give the proof for the discrete case $(t \in \mathbb{N})$. Let $P(n):=$ $P(n, z)$. We have that $\forall n \in \mathbb{N}, \exists k_{n}, p_{n} \in \mathbb{N}$, with $0 \leq p_{n} \leq N-1$, such that $n=k_{n} N+p_{n}$. Let $B_{s}:=P\left(N, z_{s N}\right), \forall s \geq 0$. Here, $z_{0}=z$. Hence $B_{s} \gg 0, \forall s \geq 0$. Let $\tilde{P}(n)=B_{k_{n}} \cdots B_{0}$. So $P(n)=P\left(p_{n}, z_{k_{n} N}\right) \tilde{P}(n)$. First, we want to apply Theorem 3.4. in [16] for the sequence of matrices $B_{0}, \ldots, B_{s}, \ldots$. Since $\overline{\mathcal{O}^{+}(z)}$ is compact, it follows that there exist constant matrices $C, D \gg 0$ such that $D \geq B_{s} \geq C, \forall s \geq 0$. Let $\delta=\min _{i, j}\left(C_{i j}\right)$ and $\gamma=\max _{i, j}\left(D_{i j}\right)$. So, the following hold:

a) $\min _{i, j}\left(B_{s}\right)_{i j} \geq \delta>0, \forall s \geq 0$;

b) $\max _{i, j}\left(B_{s}\right)_{i j} \leq \gamma<\infty$.

Thus, hypotheses of [16, Theorem 3.4] hold and (see exercise 3.6 in [16]) we have that

$$
\frac{\tilde{P}(n)_{l i}}{\tilde{P}(n)_{l j}} \rightarrow c_{i j}>0 \text { as } n \rightarrow \infty,
$$

for some $c_{i j}$ independent of $l$. Denote by $\tilde{P}(n)^{i}$ the $i^{t h}$ column of $\tilde{P}(n)$. Then (32) implies that

$$
\lim _{n \rightarrow \infty} \frac{\left|\tilde{P}(n)^{i}\right|}{\left|\tilde{P}(n)^{j}\right|}=c_{i j}
$$

Let $e_{i} \in U$ be the unit vector whose $i^{\text {th }}$ component equals one, and the other components are zero. Then, using (33), we get, for any $i \in\{1, \ldots, q\}$, that

$$
\begin{aligned}
\lambda\left(z, e_{i}\right) & =\limsup _{n \rightarrow \infty} \frac{1}{n} \ln \left|P\left(p_{n}, z_{k_{n} N}\right) \tilde{P}(n) e_{i}\right| \leq \limsup _{n \rightarrow \infty} \frac{1}{n}\left(\ln || P\left(p_{n}, z_{k_{n} N}\right) \|+\right. \\
& \left.+\ln \left|\tilde{P}(n)^{i}\right|\right)=\limsup _{n \rightarrow \infty} \frac{1}{n} \ln \left|\tilde{P}(n)^{i}\right| .
\end{aligned}
$$

On the other hand,

$$
\begin{aligned}
\lambda\left(z, e_{i}\right) & \geq \limsup _{n \rightarrow \infty} \frac{1}{k_{n} N} \ln \left|P\left(k_{n} N, z\right) e_{i}\right|=\limsup _{n \rightarrow \infty} \frac{1}{k_{n} N} \ln \left|\tilde{P}(n)^{i}\right| \\
& =\limsup _{n \rightarrow \infty} \frac{n}{k_{n} N} \frac{1}{n} \ln \left|\tilde{P}(n)^{i}\right|=\limsup _{n \rightarrow \infty} \frac{1}{n} \ln \left|\tilde{P}(n)^{i}\right| .
\end{aligned}
$$

Thus, from (34) and (35) we have that

$$
\lambda\left(z, e_{i}\right)=\limsup _{n \rightarrow \infty} \frac{1}{n} \ln \left|\tilde{P}(n)^{i}\right| .
$$

But, for any $i, j \in\{1, \ldots, q\}$ we have that 


$$
\begin{aligned}
\limsup _{n \rightarrow \infty} \frac{1}{n} \ln \left|\tilde{P}(n)^{i}\right| & =\limsup _{n \rightarrow \infty} \frac{1}{n} \ln \left(\frac{\left|\tilde{P}(n)^{i}\right|}{\left|\tilde{P}(n)^{j}\right|}\left|\tilde{P}(n)^{j}\right|\right) \\
& =\limsup _{n \rightarrow \infty}\left(\frac{1}{n} \ln \frac{\left|\tilde{P}(n)^{i}\right|}{\left|\tilde{P}(n)^{j}\right|}+\frac{1}{n} \ln \left|\tilde{P}(n)^{j}\right|\right) \\
& =\limsup _{n \rightarrow \infty} \frac{1}{n} \ln \left|\tilde{P}(n)^{j}\right| .
\end{aligned}
$$

Thus, let

$$
c=\lambda\left(z, e_{i}\right)=\limsup _{n \rightarrow \infty} \frac{1}{n} \ln \left|\tilde{P}(n)^{i}\right|, \forall i=1, \ldots, q
$$

Let $\eta \in U$. There exist $p_{1}, \ldots, p_{q} \in[0,1]$ such that $\eta=\sum_{i=1}^{q} p_{i} e_{i}$. Then, using (37), we obtain

$$
\begin{aligned}
\lambda(z, \eta) & =\limsup _{n \rightarrow \infty} \frac{1}{n} \ln |P(n) \eta|=\limsup _{n \rightarrow \infty} \frac{1}{n} \ln \left|\sum_{i=1}^{q} p_{i} P(n) e_{i}\right| \\
& \geq \limsup _{n \rightarrow \infty} \frac{1}{n} \sum_{i=1}^{q} p_{i} \ln \left|P(n) e_{i}\right|=\limsup _{n \rightarrow \infty} \frac{1}{n} \ln \left|P(n) e_{i}\right|=c .
\end{aligned}
$$

On the other hand, we have

$$
\lambda(z, \eta)=\lambda\left(z, \sum_{i=1}^{q} p_{i} e_{i}\right) \leq \max _{i=1, . ., q} \lambda\left(z, e_{i}\right)=c
$$

where we used the following two properties of Lyapunov exponents (see [1] page 114):

1) $\lambda\left(z, \eta_{1}+\eta_{2}\right) \leq \max \left\{\lambda\left(z, \eta_{1}\right), \lambda\left(z, \eta_{2}\right)\right\}$, and

2) $\lambda(z, a \eta)=\lambda(z, \eta), \forall a \in \mathbb{R} \backslash\{0\}$

From (38) and (39) we obtain $\lambda(z, \eta)=c$. It is clear that

$$
c=\lambda(z, \eta) \leq \limsup _{n \rightarrow \infty} \frac{1}{n} \ln \|P(n)\| .
$$

Now, we want to show the opposite inequality. Because all norms on a finite dimensional normed linear space are equivalent, there exist constants $a, b>0$ such that $a\|B\|_{1} \leq\|B\| \leq b\|B\|_{1}$ for all matrices $B$, where $\|B\|_{1}=\max _{i}\left|B^{i}\right|$. Then

$$
\begin{aligned}
\limsup _{n \rightarrow \infty} \frac{1}{n} \ln \|P(n)\| & \leq \limsup _{n \rightarrow \infty} \frac{1}{n} \ln \left(\left\|P\left(p_{n}, z_{k_{n} N}\right)\right\| \cdot\|\tilde{P}(n)\|\right) \\
& =\limsup _{n \rightarrow \infty} \frac{1}{n} \ln \|\tilde{P}(n)\| \\
& \leq \limsup _{n \rightarrow \infty} \frac{1}{n} \ln \left(b\|\tilde{P}(n)\|_{1}\right) \\
& =\limsup _{n \rightarrow \infty} \frac{1}{n} \ln \|\tilde{P}(n)\|_{1}=\lim _{k \rightarrow \infty} \frac{1}{n_{k}} \ln \left\|\tilde{P}\left(n_{k}\right)\right\|_{1},
\end{aligned}
$$


for some sequence $\left(n_{k}\right)_{k} \subseteq \mathbb{N}, n_{k} \rightarrow \infty$ as $k \rightarrow \infty$. There exists $j \in\{1, \ldots, q\}$ such that $\left\|\tilde{P}\left(n_{k}\right)\right\|_{1}=\left|\tilde{P}\left(n_{k}\right)^{j}\right|$ for infinitely many $k^{\prime} s$. Hence, there exists a subsequence $\left(\tilde{n}_{k}\right)_{k}$ of $\left(n_{k}\right)_{k}$ such that $\left\|\tilde{P}\left(\tilde{n}_{k}\right)\right\|_{1}=\left|\tilde{P}\left(\tilde{n}_{k}\right)^{j}\right|, \forall k$. Then, from (41) we have that

$$
\limsup _{n \rightarrow \infty} \frac{1}{n} \ln || P(n) \| \leq \lim _{k \rightarrow \infty} \frac{1}{\tilde{n}_{k}} \ln \left|\tilde{P}\left(\tilde{n}_{k}\right)^{j}\right| \leq \limsup _{n \rightarrow \infty} \frac{1}{n} \ln \left|\tilde{P}(n)^{j}\right|=c .
$$

Now, (40) and (42) imply $\lambda(z, \eta)=\limsup _{n \rightarrow \infty} \frac{1}{n} \ln \|P(n)\|$. Since $\eta \in U$ was arbitrarily chosen, the proof for the discrete case is complete.

Now let us consider the continuous case $\left(t \in \mathbb{R}_{+}\right)$. Again, considering $z \in X$ fixed, we can denote $P(t, z)$, in short, by $P(t)$. Denote by $[\cdot]: \mathbb{R} \rightarrow \mathbb{Z}$ the greatest integer function. The same argument used to prove (36) leads to

$$
\lambda(z, \eta)=\limsup _{t \rightarrow \infty} \frac{1}{[t]} \ln |P([t]) \eta| .
$$

Let $n:=[t]$. Then $\lambda(z, \eta)=\limsup _{n \rightarrow \infty} \frac{1}{n} \ln |P(n) \eta|$. Let $B_{n}:=P\left(1, z_{n}\right), \forall n \in \mathbb{N}$. Then $P(n)=B_{n-1} \cdots B_{0}$. Our hypotheses on matrix $A(z)$ guarantee that $B_{n} \gg 0, \forall n \geq 0$ (see [17, Theorem 1.1]). Now the same proof as for the discrete case, applied with $N=1$ (hence $k_{n}=n, p_{n}=0$ and $\tilde{P}(n)=P(n)$ ), carries over and leads to

$$
\limsup _{n \rightarrow \infty} \frac{1}{n} \ln |P(n) \eta|=\limsup _{n \rightarrow \infty} \frac{1}{n} \ln \|P(n)\| .
$$

But

$$
\limsup _{n \rightarrow \infty} \frac{1}{n} \ln \|P(n)\|=\limsup _{t \rightarrow \infty} \frac{1}{[t]} \ln \|P([t])\|=\limsup _{t \rightarrow \infty} \frac{1}{t} \ln \|P(t)\|
$$

Indeed, the left side is clearly less than or equal to the right hand side and the opposite inequality is obtained as in (34). This completes our proof.

Theorem 1 shows that $M$ is a uniformly weak normally repelling set provided $\lambda(z, \eta)>0$ for all $z \in M$ and all $\eta \in U$. Furthermore, under a mild hypothesis, it suffices to show $\lambda(z, \eta)>0$ for $z \in \Omega(M)=\cup_{z \in M} \omega(z)$ and $\eta \in U$. Proposition 1 gives conditions for $\lambda(z, \eta)$ to be independent of $\eta \in U$ for all $z \in M$. In this case, using (15), we see that $\lambda(z)=\lambda(\phi(s, z)), s \geq 0$ is constant on forward orbits. We assume hereafter that $\lambda(z)=\lambda(z, \eta)$ depends only on $z \in M$. As a consequence, if the hypotheses of Theorem 1 hold, and if $\Omega(M)$ consists of a finite number of periodic orbits $O_{i}, i=1,2, \ldots, p$, then it suffices to show that $\lambda\left(z_{i}\right)>0$ for some choice $z_{i} \in O_{i}, i=1,2, \ldots, p$. In this case, only finitely many exponents must be computed. See $[3,13,14]$ where $\lambda\left(z_{i}\right)$ is related to the spectral radii of a certain Floquet matrix.

According to the multiplicative ergodic theorem $[1,6]$, if $M$ is invariant and there exists an ergodic $F$-invariant Borel probability measure $\mu$ on $M$, then $\lambda(z)$ is a constant on $M$, almost surely. Unfortunately, for our results almost sure positivity of the Lyapunov exponent does not suffice. 


\section{References}

1. Arnold, L. (1998) Random Dynamical Systems. Springer-Verlag, Berlin Heidelberg

2. Ashwin, P., Buescu, J. , Stewart, I. (1996) From attractor to chaotic saddle: a tale of transverse instability. Nonlinearity 9: 703-737

3. Ferriere, R., Gatto, M. (1995) Lyapunov Exponents and the Mathematics of Invasion in Oscillatory or Chaotic Populations. Theor. Population Biol. 48: 126-171

4. Garay, B.M., Hofbauer, J. (2003) Robust Permanence for Ecological Differential Equations, Minimax, and Discretizations. SIAM J. Math. Anal. 34: 1007-1039

5. Hirsch, M.W., Smith, H.L., Zhao, X.-Q. (2001) Chain transitivity, attractivity and strong repellors for semidynamical systems. J.Dynamics and Diff. Eqns. 13: $107-131$

6. Katok, A., Hasselblatt,B. (1995) Introduction to the Modern Theory of Dynamical Systems. Cambridge University Press, New York

7. Magal, P. , Zhao, X.-Q. (2005) Global Attractors and Steady States for Uniformly Persistent Dynamical Systems. SIAM J. Math. Anal. 37: 251-275

8. Metz, J.A.J., Nisbet, R.M. , Geritz, S.A.H. (1992) How Should We Define "Fitness" for General Ecological Scenarios? Tree 7: 198-202

9. Metz, J.A.J. (2008) Fitness. Evol. Ecol. 2: 1599-1612

10. Rand, D.A., Wilson, H.B., McGlade, J.M. (1994) Dynamics and Evolution: Evolutionarily Stable Attractors, Invasion Exponents and Phenotype Dynamics. Philosophical Transactions: Biological Sciences 343: 261-283

11. Salceanu, P.L., Smith, H.L. (2009) Persistence in a Discrete-time, Stagestructured Epidemic Model. J. Difference Equ. Appl. to appear

12. Salceanu, P.L. , Smith, H.L. (2009) Persistence in a Discrete-time Stagestructured Fungal Disease Model. J. Biol. Dynamics 3: 271-285

13. Salceanu, P.L., Smith, H.L. (2009) Lyapunov Exponents and Persistence in Discrete Dynamical Systems. Discrete and Continuous Dynamical Systems-B to appear

14. Salceanu, P.L. (2009) Lyapunov exponents and persistence in dynamical systems, with applications to some discrete-time models. Phd. Thesis, Arizona State University

15. Schreiber, S.J. (2000) Criteria for $C^{r}$ Robust Permanence. J. Differ. Equations 162: $400-426$

16. Seneta, E. (1973) Non-negative Matrices, an Introduction to Theory and Applications. Halsted Press, New York

17. Smith, H.L. (1995) Monotone Dynamical Systems: an introduction to the theory of competitive and cooperative systems. Amer. Math. Soc. Surveys and Monograghs 41

18. Smith, H.L., Zhao, X.-Q. (2001) Robust Persistence for Semidynamical Systems. Nonlinear Anal. 47: 6169-6179

19. Thieme, H.R. (2003) Mathematics in Population Biology. Princeton University Press, New Jersey

20. Zhao, X.-Q. (2003) Dynamical Systems in Population Biology. Springer-Verlag, New York 